\title{
Importancia de la Aviación Naval contra el narcotráfico en los mares de Colombia ${ }^{1}$
}

\author{
https://doi.org/10.21830/9789585380226.04
}

\author{
Christian Acevedo Navas ${ }^{2}$ \\ Escuela Militar de Cadetes "General José María Córdova” \\ Wilmer Andrés Diaz Blanco ${ }^{3}$ \\ Armada Nacional
}

\begin{abstract}
Resumen. Este capítulo analiza la importancia de la Aviación Naval de Colombia en la lucha contra el narcotráfico en el mar. Se desarrolla un ensayo reflexivo teórico, mediante análisis de contenido de literatura especializada sobre relaciones internacionales, estrategia marítima, estrategia nacional, estrategia militar, política y estrategia, poder naval, poder marítimo, oceanopolítica y mar presencial. Se revisa el marco jurídico y de políticas públicas nacionales e internacionales frente al narcotráfico, así como algunas cifras recientes sobre este ilícito. A partir de los elementos revisados, se concluye que el narcotráfico es una amenaza a la seguridad nacional tanto de Colombia como del sistema internacional y que, en este sentido, la Aviación Naval cumple un papel fundamental no solo mediante la disuasión y la interdicción, sino también mediante su aporte al control de la soberanía, a la estabilidad política y económica nacional, y al fortalecimiento de las relaciones de cooperación con otras naciones.
\end{abstract}

Palabras clave: Aviación Naval; defensa; estrategia marítima; estupefaciente; narcotráfico; poder naval; relaciones internacionales.

1 Este capítulo de libro se deriva parcialmente del desarrollo de una tesis de maestría, a cargo de uno de los autores. No recibe financiación externa diferente del propio pecunio del autor. Los puntos de vista y los resultados presentados en este capítulo pertenecen a los autores y no reflejan necesariamente los de las instituciones participantes.

2 Doctor en Ciencias Sociales de la Universidad del Norte. Profesional en Ciencias Navales de la Escuela Naval de Cadetes "Almirante Padilla". Asesor de investigaciones de la Facultad de Administración de la Escuela Militar de Cadetes "General José María Córdova” (EsMIC), Bogotá, D. C., Colombia. ORCID: https://orcid.org/0000-0003-4880-3024 - Contacto: christian.acevedo@esmic.edu.co

3 Comandante Gataf de la Armada Nacional, Colombia. Magíster en Relaciones Internacionales de la Universidad Militar Nueva Granada. Administrador de la Escuela Naval de Cadetes "Almirante Padilla”. Orcid: https://orcid.org/0000-0003-1865-0012 - Contacto: andres.diaz@armada.mil.co 


\section{Introducción}

El narcotráfico impacta política, social y económicamente al sistema internacional. El narcotráfico financia los Grupos Delincuenciales Organizados, que están dispersos en el territorio y vinculados a empresas, economía y política (Cajiao et al., 2018). En este escenario, el mar colombiano se ha convertido en el espacio por donde fluye la mercancía ilícita. Por esto, el Estado colombiano debe fortalecer las estrategias para la lucha contra el narcotráfico en altamar y en los litorales. La Armada Nacional contribuye a la defensa de la nación mediante el empleo efectivo de un poder naval flexible en los espacios marítimo, fluvial y terrestre bajo su responsabilidad (Comisión Colombiana del Océano, 2018; Armada Nacional, 2021), negando el uso del mar a los Grupos Delincuenciales Organizados. Con este fin, la Aviación Naval desempeńa un importante papel, con medios aeronavales disuasivos, aportando al poder naval como una extensión de los sensores de las unidades de superficie. El objetivo del presente trabajo consiste en analizar la importancia de la Aviación Naval en la lucha contra el narcotráfico en los mares de Colombia.

\section{Métodos}

Este trabajo consiste en un ensayo reflexivo teórico, a partir de una revisión bibliográfica. Los documentos funcionan como referentes teórico-conceptuales para la reflexión y no como datos en sí mismos. En cuanto a su alcance, el ejercicio es descriptivo. Respecto a su naturaleza, es un ejercicio analítico. Desde la óptica temporal, se trata de un trabajo transversal. Los conceptos de población y muestra no aplican para ser definidos, toda vez que en este diseño los que serían datos empíricos secundarios son los referentes documentales sobre los cuales se hace el análisis. Estos documentos fueron seleccionados por la relación con las temáticas, la relevancia en cuanto a las citaciones que las respaldan y la conveniencia de su disponibilidad. En cuanto al método, se desarrolló un análisis de contenido, en el cual se revisaron los referentes teóricos y los documentos mencionados, a partir de los cuales se extrajo los elementos claves (significados, afirmaciones determinantes y cate- 
gorías), que fueron analizados mediante la ayuda de una matriz de análisis. Esta matriz permitió plantear las discriminaciones y las selecciones descriptivas y relacionales, así como la coherencia interna de cada documento consultado (análisis vertical) y la coherencia conceptual para las categorías definidas entre todos los documentos consultados (análisis horizontal).

\section{Resultados y discusión}

En esta sección se presentan los principales hallazgos y reflexiones derivadas del análisis de contenido que se efectuó. Se revisa el tema del narcotráfico en el mar a la luz de las relaciones internacionales, la estrategia marítima y el marco jurídico y de políticas públicas.

\section{Teorías de las relaciones internacionales y el narcotráfico}

En el campo de las relaciones internacionales se entiende que a través del Estado se desarrollan las relaciones políticas, sociales, económicas y de poder. Cada Estado busca cumplir sus objetivos mediante instrumentos jurídicos y diplomáticos (Vázquez \& González, 2015) y tiene derecho a defender su soberanía, entendida como su supremacía, capacidad para relacionarse con otros y organizar su política, economía y leyes sin intervención de terceros (Rosales, 2005). Colombia se ajusta a la definición de Estado, pues cuenta con población, territorio y poder (García, 2002).

Para el realismo, el Estado es central en las relaciones internacionales. Las relaciones se basan en poder, particularmente militar (Morguenthau \& Thompson, 1986). Los Estados se acomodan a una jerarquía en sus interacciones. Por su parte, el neorrealismo parte del realismo, pero agrega nuevos elementos: Waltz (1988) entiende las relaciones internacionales como un proceso racional, moldeado por los mercados. Para Sodupe (2003), el neorrealismo es la sistematización del realismo, de manera que funciona como una estructura de unidades en interacción. En este escenario, la teoría de la interdependencia compleja incluye tres rasgos: canales múltiples de conexión entre sociedades, agenda diversa y no jerarquizada de relaciones entre los Estados y el aparato militar que se usa con diversos propósitos, no solo en contra de otros Estados (Keohane \& Nye, 1988). 
Ratzel (2011) aclara que en geografía política no se contemplan los buques de guerra como parte flotante del Estado, ni la proyección del territorio hacia el cielo, aunque reconoce la importancia de los mares adyacentes, de modo que la soberanía se traslapa entre naciones. Específicamente en Colombia, la producción de coca traspasa las fronteras, de manera que es una amenaza transnacional. Los narcotraficantes utilizan el mar colombiano, lo cual obliga al Estado a mantener su control y minimizar el impacto en otras naciones. Al respecto, la teoría del espacio vital considera los Grupos Delincuenciales Organizados narcotraficantes como actores, igual que los Estados, debido a su impacto transnacional (Haushofer, 2013).

Desde la postura del realismo, el narcotráfico es una amenaza para los Estados, y esto justifica la política de seguridad. Desde el neorrealismo, la globalización del narcotráfico es el efecto más nocivo. El mercado de las drogas se ha visto facilitado por los Estados, que no lo han controlado completamente en el mar (Sodupe, 2003). El neorrealismo asume que los Estados con menor poder forman parte de una estructura, en la cual hay posiciones recíprocas. Las interacciones en términos realistas se verían restringidas si se desarrollaran de forma individual y directa, de modo que si la lucha contra el narcotráfico fuese solo de Colombia, no produciría efecto. En este sentido, esta estructura hace necesario que los países cooperen como iguales y distribuyan sus capacidades (Sodupe, 2003). Desde la interdependencia compleja, además de la economía, ya prevista por el neorrealismo, aparecen factores como las comunicaciones, las aspiraciones humanas, la tecnología, los intercambios sociales y también los Grupos Delincuenciales Organizados (Keohane \& Nye, 1988).

\section{Estrategia marítima y narcotráfico}

La estrategia es el uso del combate para los fines de la guerra, en la que todo acto bélico debe tener un propósito conectado al objetivo superior (Clausewitz, 1832; citado por Uribe et al., 2016). La estrategia marítima es parte de la estrategia nacional, y trata de la creación, mantenimiento y empleo del poder marítimo del Estado para promover y proteger sus intereses marítimos (Uribe et al., 2016). Los intereses marítimos se refieren a valores económicos y sociales. El poder naval se refiere a valores políticos y militares asociados 
a la protección del mar, mientras que la conciencia marítima complementa y multiplica los intereses marítimos y el poder naval (Terzago, 2005). Por su parte, la oceanopolítica define estrategias para que el mar sea un espacio de progreso (Martínez, 1993).

La lucha contra las drogas en el mar requiere un planeamiento similar a la guerra, exige preparar encuentros, interdicción en este caso, para neutralizar la amenaza. La estrategia marítima comprende fines, medios, modos, riesgos, estrategia del adversario y entorno (Uribe et al., 2016). El adversario son los Grupos Delincuenciales Organizados narcotraficantes, cuya estrategia consiste en el aprovechamiento del mar mediante lanchas Go-fast, embarcaciones de carga, pesca o recreo, y motonaves de bajo perfil, entre otros medios (Centro Internacional Marítimo de Análisis Contra el Narcotráfico [Cimcon], 2018b). En el 2017, la modalidad más frecuente fue la lancha Go-fast (24,1\%), seguida por contenedores $(23,5 \%)$ y embarcaciones de pesca artesanal (14,2\%). En el 2018, la Armada Nacional aumentó en $5 \%$ las incautaciones, gracias a la cooperación con marinas de la región, a pesar de un descenso del $26 \%$ en el número de interdicciones. Es decir, se detuvieron menos lanchas, pero se incautó más mercancía. En el 2018 se incautaron 127 toneladas de cocaína, de 1.874 toneladas estimadas producidas, lo cual significa que se logró una afectación de solo el 6,7 \% al ilícito (Cimcon, 2018a). En tal sentido, el adversario, en este caso los Grupos Delincuenciales Organizados, se convierte en una amenaza a la seguridad y la defensa del Estado, de la región y del mundo (Meléndez, 2019; Uribe et al., 2016).

Para combatir esta amenaza se deben considerar las interrelaciones entre los cuatro niveles de la estrategia: político, estratégico, operacional y táctico. Debe haber una relación entre política y estrategia, en la cual la estrategia militar esté bajo consideraciones políticas del Estado. El comandante militar considera los objetivos políticos frente a la autonomía que requiere en sus acciones contra el narcotráfico. Otro nivel de relación se da entre estrategia y táctica, en la cual las acciones tácticas se realizan para satisfacer la estrategia y no al contrario. El nivel operacional enlaza los objetivos estratégicos con sus correspondientes acciones tácticas (Uribe et al., 2016). 
En este escenario, la Ruta Futuro 2018 estableció como uno de sus objetivos intensificar la interdicción para limitar la salida de droga a los mercados (Ministerio de Justicia y del Derecho, 2018). Este objetivo de nivel político está alineado con la Política de Defensa y Seguridad 2019, que garantiza la soberanía nacional, la integridad territorial, la independencia y la lucha contra el crimen transnacional (Ministerio de Defensa, 2019). Por su parte, el Plan Estratégico Militar 2030 buscar ser un referente internacional en la lucha contra las amenazas transnacionales (Comando General de las Fuerzas Militares [CGFM], 2015). Asimismo, el Plan Estratégico Naval establece que la Armada Nacional concentrará toda su capacidad en contribuir a la consolidación de la Política de Defensa y Seguridad, así como en eliminar la amenaza narcoterrorista (Armada Nacional, 2020). Así, el Estado colombiano alinea sus objetivos y planes contra las drogas en los niveles político, estratégico, operacional y táctico.

El poder marítimo tiene unos medios, consistentes en las capacidades marítimas militares y civiles, armadas, industrias marítimas y navales, guardacostas e incluso fuerzas terrestres y aéreas. Además, tiene unos fines, entre ellos la capacidad de influir desde el Estado en el comportamiento de terceros frente al mar. Por supuesto, algunos países tienen mayor poder marítimo que otros (Till, 2007), el cual se conforma de dos elementos: los intereses marítimos y el poder naval, al que se le suma la conciencia marítima. Los intereses marítimos son las aspiraciones del Estado para usar y beneficiarse del mar, entre ellos: poder naval; transporte y comercio marítimo; recursos ambientales marino-costeros; educación marítima; soberanía e integridad del territorio marítimo nacional; investigación científica, tecnológica y de innovación; conciencia, apropiación territorial y cultura marítima; turismo marítimo y recreación; seguridad integral marítima, y ordenamiento marino-costero (PNoec, 2018).

En este contexto, la Aviación Naval tiene una importante participación, teniendo en cuenta que los medios aeronavales son primordiales para proteger los intereses marítimos y el territorio nacional, pues pueden sobrevolar los mares nacionales y las aguas internacionales adyacentes. También genera conciencia sobre la amenaza transnacional del narcotráfico, crea apropiación territorial al tener presencia permanente, desplazarse rápidamente y 
cubrir rangos superiores de patrullaje; proteger recursos marino-ambientales; controlar la pesca ilegal o la explotación ilícita, y hacer interdicción marítima, entre otras operaciones (González, 1989).

La conciencia marítima incluye el conocimiento del mar, su naturaleza y las posibilidades económicas, militares, políticas y sociales. Para el caso colombiano, la geografía debería facilitar la conciencia marítima de la nación para convertirse en potencia marítima (Terzago, 2005) o por lo menos en una Potencia Media Oceánica [PMO]. Una PMO es un Estado con amplias capacidades de poder marítimo, que gestiona integralmente su territorio, y se proyecta internacionalmente, a fin de mejorar el bienestar ciudadano, propiciar el desarrollo sostenible e influir en la región (PNOEC, 2018).

El poder naval es el componente militar de un Estado y su armada, que protege los intereses marítimos (Terzago, 2005). Se compone de dos elementos: fuerza y posición estratégica, que se unen y activan por la voluntad estratégica. La fuerza, de naturaleza estratégica, está compuesta por la armada y sus capacidades (Uribe et al., 2016). La posición estratégica, de orden geográfico, es punto de partida y apoyo para las operaciones. La constituyen bases navales e instalaciones de apoyo logístico, que permiten alcanzar los objetivos de guerra o lucha antidrogas en el mar. La fuerza y la posición geográfica necesitan de la voluntad estratégica, que dinamiza el poder naval. La posición geográfica es inerte y lo que le da vida es la fuerza, que, impulsada por la voluntad estratégica, se vale de su localización y medios de apoyo para las operaciones.

Desde la oceanopolítica, el territorio oceánico va mucho más allá de las 200 millas de Zona Económica Exclusiva, e incluye el mar territorial, el mar patrimonial y el mar presencial. Este último es aquel hasta donde el Estado tiene capacidad de permanecer, más allá de la Zona Económica Exclusiva, para proteger sus intereses. Así, es determinante la actividad de la Armada en el mar, para prevenir la afectación a la seguridad y patrimonio nacionales (Dávalos, 2018; Martínez, 1993).

\section{Marco legal de políticas públicas y narcotráfico}

La Convención de 1961 sobre estupefacientes, enmendada por el Protocolo de 1972, y la Convención de 1988 promueven la cooperación inter- 
nacional en la lucha contra las drogas (Oficina de Naciones Unidas contra la Droga y el Delito [Unodc], 1988; Onu, 1961). En el 2016, la OnU aprobó la Asamblea General de las Naciones Unidas [UnGAss, por su sigla en inglés) y se definió una estrategia de apoyo a la lucha contra las drogas (Ministerio de Justicia y del Derecho, 2017).

En el plano nacional, Colombia cuenta con una serie de normas: los Lineamientos de la Política Nacional de Drogas (Ministerio de Justicia y del Derecho, 2017); el Programa Nacional Integral de Sustitución; la Reforma Rural Integral; la Ruta Futuro (Ministerio de Justicia y del Derecho, 2018) y la Política de Defensa y Seguridad, entre otras estrategias (Ministerio de Defensa, 2019). También se aprobó la Política Nacional del Océano 20162030 (Comisión Colombiana del Océano, 2018) y el Centro Internacional Marítimo de Análisis Contra el Narcotráfico [CimCon]. En la normatividad se exhorta a la contribución del Estado colombiano para la prevención y el control del narcotráfico.

La normatividad parte de la Convención de 1961, en la que se reconoce que las drogas afectan gravemente al ser humano, son un peligro social y económico, y es una obligación prevenir y luchar contra este delito (ONU, 1961). A partir de esto, el Congreso de la República aprobó la Convención de 1961 y el Protocolo de 1972 (Ley 13 de 1974). Los firmantes deben asegurar medidas preventivas y represivas contra este tráfico ilícito, de modo que la Armada Nacional, con su Aviación Naval, no solo es una fuerza represiva para la captura de narcotraficantes en altamar, sino también preventiva y disuasiva al tener medios aeronavales volando sobre el mar e impidiendo su uso para el delito.

La Convención de 1988 establece que para naves sospechosas se podrá solicitar asistencia de terceras partes, que prestarán los medios, y se podrá autorizar abordaje, inspección u otras medidas, solamente aplicadas por buques de guerra o aeronaves militares (UNODC, 1988), de manea que esto exige la participación de la Armada Nacional y la Aviación Naval. La Comisión de Estupefacientes de las Naciones Unidas (CND, por su sigla en inglés), que incluye a Colombia, fue creada en 1946 con el fin de asistir a la ONU en la verificación de tratados sobre drogas. Posteriormente, en 1991, la ONU amplió 
el mandato de esta comisión, con el objetivo de que funcionara como órgano rector de la UNODC (CND, 1946).

La UNGASs 2016 se realizó como un proceso para examinar los progresos en la aplicación de la declaración de la política y el plan de acción sobre cooperación internacional, de tal modo que permitiera consolidar una estrategia integral para la lucha mundial contra las drogas, con lo cual consolidó el requerimiento de revisar las políticas públicas para reducir el narcotráfico y su afectación en la sociedad (Asamblea General de las Naciones Unidas, 2016).

Por su parte, la Ruta Futuro propone el ataque a la oferta de drogas y las rentas del crimen organizado. Específicamente, con su política nacional de drogas el Estado colombiano busca responder al tráfico ilícito orientando sus esfuerzos para impactarlo, para lo cual prioriza la interdicción (Ministerio de Justicia y del Derecho, 2018). En el Programa Nacional Integral de Sustitución se suspendió la aspersión aérea, con el objetivo de resolver el fenómeno de las drogas en conjunto con las comunidades. En la Reforma Rural Integral se define la transformación de las condiciones de vida de las comunidades rurales mediante la superación de la pobreza y la brecha campo-ciudad (Alta Consejería para el Posconflicto, 2017).

En la Política de Defensa y Seguridad, se destaca que las amenazas transnacionales se han agudizado para Colombia y que las drogas ilícitas financian los Grupos Delincuenciales Organizados (Ministerio de Defensa, 2019). La Aviación Naval puede cumplir funciones disuasivas que aporten al logro de los objetivos estratégicos y las líneas de política, garantizando la soberanía, la independencia y la integridad territorial. La Política de Defensa y Seguridad también prevé la adopción de una estrategia de seguridad fronteriza a partir de planes binacionales, interoperabilidad y operaciones combinadas. La Armada Nacional, con la Aviación Naval, puede formar parte de esta estrategia con los planes ya existentes y con nuevas operaciones sumadas a esfuerzos con países vecinos. La Política de Defensa y Seguridad también establece el control institucional del territorio, en el cual la Armada Nacional continúa teniendo la responsabilidad del espacio marítimo.

La Política Nacional del Océano y de los Espacios Costeros 2016-2030 es referente para la proyección de Colombia como PMO. Este documento prevé 
como determinante la participación del poder naval, que como se ha recalcado, incluye a la Aviación Naval como factor clave en la lucha contra el narcotráfico. El Plan Estratégico Militar 2030 establece elementos para modernizar y fortalecer las Fuerzas Militares (CGFM, 2015). El Plan Estratégico Naval 2020 establece que será empleado el poder naval para contribuir al logro de los objetivos políticos y militares que garanticen los intereses nacionales. Señala como prioridad el triunfo sobre el narcoterrorismo y el desarrollo de esfuerzos institucionales para el cumplimiento de la misión. La Armada Nacional concentrará toda su capacidad en contribuir a la consolidación de la Política de Defensa y Seguridad, eliminando la amenaza narcoterrorista. También sostiene que el desafío terrorista constituye un reto para la Armada Nacional, pues implica reorientar el empleo de los medios para generar fortalezas frente a un enemigo asimétrico y así contribuir significativamente con las capacidades humanas y tecnológicas de la institución a dar un vuelco estratégico al desarrollo del conflicto (Armada Nacional, 2020).

\section{Conclusiones}

Con base en el análisis presentado en las secciones anteriores, creemos que lo revisado en este capítulo evidencia la importancia de la Aviación Naval colombiana en la lucha contra el narcotráfico en el mar. Por una parte, se observa que el incumplimiento en el control del mar por parte del Estado colombiano puede deteriorar las relaciones internacionales, de modo que la lucha contra el narcotráfico en el mar y la interdicción marítima forman parte de los objetivos de política exterior colombiana. Creemos que es obligación del Estado defender su soberanía territorial con poder militar y naval, incluida la Aviación Naval, así como afectar las finanzas de los Grupos Delincuenciales Organizados, perspectiva que se soporta en el realismo y el neorrealismo.

También reivindicamos que, desde la estrategia marítima, la Aviación Naval apoye la lucha contra el narcotráfico en altamar como parte de la estrategia nacional y marítima, en cumplimiento de los objetivos políticos, estratégicos, operacionales y tácticos. Finalmente, desde la óptica normativa hemos evidenciado una plataforma suficiente y coherente de legalidad para la lucha contra el narcotráfico de toda la institucionalidad y, de forma derivada 
y consecuente, por parte de la Aviación Naval. Esta plataforma va desde la Convención de 1961, pasando por la Convención de 1988, la Comisión de Estupefacientes y la UNGASS 2016 en la esfera internacional, hasta la Ruta Futuro, los Lineamientos de la Política Nacional de Drogas, la Política de Defensa y Seguridad 2019, la Política Nacional del Océano y de los Espacios Costeros, el Plan Estratégico Militar 2030 y el Plan Estratégico Naval, en el ámbito nacional.

\section{Referencias}

Alta Consejería para el Posconflicto. (2017). Reforma Rural Integral. Presidencia de la República.

Armada Nacional. (2020). Lineamiento estratégico. https://www.armada.mil.co/es/content/6-planeacion

Armada Nacional. (2021). Misión y visión de la Armada Nacional. https://www.armada.mil.co/ es/content/mision-y-vision-armada-nacional

Asamblea General de las Naciones Unidas [UNGASS]. (2016). Proyecto de Resolución "Nuestro compromiso conjunto de abordar y contrarrestar eficazmente el problema mundial de las drogas". ONU.

Cajiao, A., González, P., Pardo, D., \& Zapata, O. (2018). Una aproximación al crimen transnacional organizado: Redes de narcotráfico Colombia-España. Real Instituto Elcano.

Centro Internacional Marítimo de Análisis Contra el Narcotráfico [CIMCON]. (2018a). Dinámica del narcotráfico marítimo en Colombia y la región año 2017. Armada Nacional.

Centro Internacional Marítimo de Análisis Contra el Narcotráfico [CIMCON]. (2018b). Informe anual de narcotráfico 2018. Armada Nacional.

Comando General de las Fuerzas Militares [CGFM]. (2015). Plan Estratégico Militar 2030. https://issuu.com/fuerzasmilitaresdecolombia/docs/plan_estrategico_militar_2030_esp

Comisión Colombiana del Océano. (2018). Política Nacional del Océano y de los Espacios Costeros.

Comisión de Estupefacientes de las Naciones Unidas [CND]. (1946). United Nations Comission on Narcotic Drugs. https://www.unodc.org/unodc/es/commissions/CND/ index.html

Dávalos, J. (2018). Oceanopolítica. El mar como imperativo de desarrollo y seguridad. ESPE.

García, E. (2002). Introducción al estudio del derecho. Porrúa.

González, E. (1989). La aviación naval: Su dimensión y empleo. Revista de Marina, 6(89), 595-613. https://revistamarina.cl/revistas/1989/6/egonzalezr.pdf

Haushofer, K. (2013). Los fundamentos geográficos de la política exterior. Geopolítica(s), Revista de Estudios sobre Espacio y Poder, 3(2), 329-336. 
Keohane, R., \& Nye, J. (1988). Poder e interdependencia: La politica mundial en transición. Grupo Editor Latinoamericano.

Ley 13. (1974). Por medio de la cual se aprueba la Convención Única sobre Estupefacientes, hecho en Nueva York el 30 de marzo de 1961, y su Protocolo de Modificaciones, hecho en Ginebra el 25 de marzo de 1972. Diario Oficial, n. ${ }^{\circ}$ 34228. Congreso de la República.

Martínez, J. (1993). Oceanopolitica: Una alternativa para el desarrollo. Andrés Bello.

Meléndez, J. (2019). Los escenarios institucionales de la defensa nacional en Nicaragua. RESDAL.

Ministerio de Defensa. (2019). Politica de Defensa y Seguridad (PDS). https://www.mindefensa.gov.co/irj/go/km/docs/Mindefensa/Documentos/descargas/Prensa/Documentos/ politica_defensa_deguridad2019.pdf

Ministerio de Justicia y del Derecho. (2017). Lineamientos Política Nacional de Drogas. Dirección de Política de Drogas y Actividades Relacionadas. http://www.odc.gov.co/ Portals/1/publicaciones/pdf/pnacional/PN031152017_lineamientos_politica_nacional_ drogas_2017.pdf

Ministerio de Justicia y del Derecho. (2018). Ruta Futuro: Politica integral para enfrentar el problema de las drogas 2018. http://www.odc.gov.co/Portals/1/Docs/POLITICA_ RUTA_FUTURO_ODC.pdf

Morguenthau, H., \& Thompson, K. (1986). Politica entre las naciones: La lucha por el poder y la paz. Grupo Editor Latinoamericano.

Oficina de las Naciones Unidas contra la Droga y el Delito [UNODC]. (1988). Convención de las Naciones Unidas contra el Tráfico Ilícito de Estupefacientes y Sustancias Sicotrópicas. https://www.unodc.org/pdf/convention_1988_es.pdf

Organización de las Naciones Unidas [ONU]. (1961). Convención Única de 1961 sobre estupefacientes. https://www.incb.org/documents/Narcotic-Drugs/1961-Convention/convention_1961_es.pdf

Ratzel, F. (2011). Las leyes del crecimiento espacial de los Estados: Una contribución a la geografía política científica. Geopolitica(s), Revista de Estudios sobre Espacio y Poder, 2(1), 135-156.

Rosales, G. (2005). Geopolitica y geoestrategia, liderazgo y poder. Ensayos. UMNG.

Sodupe, K. (2003). La teoría de las relaciones internacionales a comienzo del siglo XXI. Universidad del País Vasco.

Terzago, J. (2005). Alfred Thayer Mahan [1840-1914], Contraalmirante U.S. Navy, su contribución como historiador, estratega y geopolítico. Universidad Viña del Mar.

Till, G. (2007). Poder maritimo: Una guia para el siglo XXI. Instituto de Publicaciones Navales del Centro Naval.

Uribe, S., Díaz, J., \& Rodríguez M. (2016). Estrategia marítima, evolución y prospectiva. Escuela Superior de Guerra.

Vázquez, J., \& González, S. (2015). El Estado y las relaciones internacionales. En X. Arango, \& A. Hernández (Eds.), Ciencia politica: perspectiva multidisciplinaria (pp. 223-249). Tirant lo Blanch.

Waltz, K. (1988). The origins of war in Neorealist Theory. The Journal of Interdisciplinary History, 18(4), 615-628. 